\title{
多通道运动特征融合的微表情识别方法
}

\author{
余文祥 1,2$)$, 刘斌 ${ }^{2)}$, 陶建华 ${ }^{1,2)^{*}}$, 张昊 ${ }^{1,2)}$, 吕钊 ${ }^{1)}$ \\ 1) (安徽大学多模态认知计算安徽省重点实验室 合肥 230601) \\ 2) (中国科学院自动化研究所模式识别国家重点实验室 北京 100190) \\ (jhtao@nlpr.ia.ac.cn)
}

\begin{abstract}
摘 要: 针对已有的微表情识别中由于微表情变化幅度不明显, 导致细微特征容易在学习过程中丢失, 从而使模型的性 能受到限制的问题, 提出一种基于运动特征的微表情识别方法. 首先分析变化幅度相对明显的区域对微表情识别的影响, 根据生理学研究对微表情变化相对活跃区域进行局部切割, 并使用并行神经网络分别对局部区域和全局区域提取特征; 然后采用一种能够提取特征级运动信息的运动特征提取模块从空间特征图中学习到运动特征, 将运动特征和空间特征进 行聚合, 以减少细微特征的丢失; 最后将局部特征和全局的聚合特征组合成新的混合特征用于微表情识别. 实验结果表 明, 在 MEGC 2019 数据集(包含 CASME II, SMIC 和 SAMM)上，未加权 $F_{1}$ 分数和未加权平均召回率的结果分别为 $81.81 \%$ 和 79.01\%, 与 MEGC 2019 最好的方法相比分别提高了 $2.96 \%$ 和 $0.77 \%$, 该方法具有更好的识别性能.
\end{abstract}

关键词：微表情识别; 动作特征提取; 神经网络; 局部特征; 特征聚合

中图法分类号: TP391.41 DOI: 10.3724/SP.J.1089.2021.18725

\section{Aggregation of Motion Features of Multiple Paths for Micro-Expression Recognition}

\author{
She Wenxiang ${ }^{1,2)}$, Liu Bin ${ }^{2)}$, Tao Jianhua ${ }^{1,2)^{*}}$, Zhang Hao ${ }^{1,2)}$, and Lyu Zhao ${ }^{1)}$ \\ 1) (Anhui Province Key Laboratory of Multimodal Cognitive Computation, Anhui University, Hefei 230601) \\ ${ }^{2)}$ (National Laboratory of Pattern Recognition, Institute of Automation, Chinese Academy of Sciences, Beijing 100190)
}

\begin{abstract}
Since the change of micro-expression is not obvious, subtle features are easily lost in the learning process, which limits the performance of the model. A micro-expression recognition method is proposed based on motion features. Firstly, in order to explore the influence of regions with obvious change on micro-expression recognition, the relatively active regions of micro-expression are locally cut according to physiological research, and the features of local region and global region are extracted respectively by using parallel neural network. When extracting features, a motion feature extraction module which can extract feature level motion information is adopted. The module can learn motion features from spatial feature graph, and aggregate motion features and spatial features to reduce the loss of subtle features. Finally, the local aggregate features and global aggregate features are combined to form a new hybrid feature for micro-expression recognition. Experiments on the MEGC 2019 (including CASME II, SMIC, and SAMM) databases show that the results of unweighted $F_{1}$ score and unweighted average recall are $81.81 \%$ and $79.01 \%$, which are improved by $2.96 \%$ and $0.77 \%$ respectively compared with the best method of MEGC 2019.
\end{abstract}

收稿日期：2020-10-19; 修回日期：2021-01-25. 基金项目：国家重点研发计划(2017YFB1002804); 国家自然科学基金(61831022, 61771472, 61773379, 61901473); 模式识别国家重点实验室开放课题基金(202000026). 余文祥(1995一), 男, 硕士研究生, 主要研究方 向为情感计算; 刘斌(1984一), 男, 博士, 副研究员, 主要研究方向为情感计算、音频信号处理; 陶建华(1972一), 男, 博士, 研究员, 博士生导师, CCF 会员, 论文通讯作者, 主要研究方向为语音合成、情感计算、模式识别; 张昊(1997一), 男, 硕士研究生, 主要研究 方向为情感计算; 吕钊(1979一), 男, 博士, 教授, 博士生导师, CCF 会员, 主要研究方向为智能信息处理、人机交互技术. 
Key words: micro-expression recognition; action feature extraction; neural network; local features; feature aggregation

面部表情是人们在交流过程中一个重要的视 觉信号, 分为宏表情和微表情. 与宏表情不同, 微 表情是一种面部肌肉变化微弱且持续时间极短的 面部活动 ${ }^{[1]}$. 相关的心理学研究 ${ }^{[2]}$ 表明, 微表情持 续时间仅为 $1 / 25 \sim 1 / 2 \mathrm{~s}$, 通常会在人们试图隐藏自 己真实情感时无意识地流露出来, 难以伪装, 可以 为揭示人类真实情感提供重要的视觉线索. 基于 这些特点, 微表情在许多领域具有重要的、潜在的

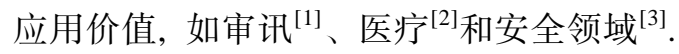

近年来, 微表情识别受到越来越多的关注, 如 何从微表情视频片段中提取微表情特征成为关键问 题. 目前, 微表情识别方法分为基于传统手工特征 的微表情识别和基于深度学习的微表情识别 2 种. 基于传统特征的方法, 如 LBP-TOP ${ }^{[4]}, \mathrm{Bi}-\mathrm{WOOF}^{[5]}$ 等, 会依赖研究人员的个人经验, 往往得到的特征 不是最优的 ${ }^{[6]}$, 在识别精度上很难有进一步的提 升. 随着深度学习在计算机视觉领域取得重大成 功, 文献[7-11]开始将深度学习的方法应用到表情 识别中, 并取得优于传统方法的结果. 因此, 本文 结合深度学习对微表情进行识别.

微表情是一个动态变化的面部表情, 且面部 变化幅度小, 利用神经网络很难从微表情帧中提 取到有效的空间特征, 因此有必要结合微表情运 动特征进行识别. 目前, 序列的运动特征提取采用 光流 ${ }^{[12]}$ 或三维神经网络(3D convolutional neural networks, 3DCNN $)^{[13]}$, 其中, 光流的计算既费时又 需要额外存储; 而 3DCNN 需要增加网络的参数 量, 在小样本数据集学习中很容易造成过拟合.

相对于宏表情，微表情还具有区域依赖性，即 它出现在面部的局部区域中 ${ }^{[14]}$. 例如, 高兴集中 在嘴部和眼部(嘴角向两侧拉升, 眼睑收缩), 惊讶 体现在嘴部和眉毛部分(嘴唇微张, 眉毛上扬), 这 些变化相对活跃的区域被称为感兴趣区域(region of interest, RoI). 在微表情识别工作中, RoI 能够避 免与微表情无关或弱相关区域带来的噪声干扰. Ekman 等 ${ }^{[15]}$ 开发了面部动作编码系统, 根据单个 肌肉或一群肌肉的运动定义不同的动作单位 (action units, AUs). Happy 等 ${ }^{[16]}$ 根据 AUs 发生频次 划分 RoI, 并且通过提取 RoI 特征对面部表情进行 识别, 其结果在 CK+数据集 ${ }^{[17]}$ 和 JAFFE 数据集 ${ }^{[18]}$ 上表现良好. 同时, Ringeval 等 ${ }^{[19]}$ 也选择了左眼+ 眉毛、右眼+眉毛和嘴巴 3 个局部区域作为 RoI 对
面部特征分析, 并取得成功. 微表情具有区域依赖 性, 通过对 RoI 有针对性的学习同样可以提高识别 性能. Yan 等 ${ }^{[20]}$ 在微表情识别工作中发现了眉毛和 嘴巴是最富表现力的 2 个部位, 这些区域在面部的 所有其他区域中贡献了大部分且有意义的微表情 细节. Wang 等 ${ }^{[21]}$ 使用特定的面部区域对微表情识 别并取得了一定成功.

针对以上问题, 本文利用深度学习构建微表 情识别框架, 采用神经网络对微表情序列进行空 间特征的提取. 为了避免额外的计算或网络参数 的增加, 框架中使用一种运动特征提取模块对空 间特征图时间通道编码运动特征, 可以像光流法 一样提取到明显的边缘特征, 更加关注动作交互 的部分. 此外, 为了研究局部信息对微表情识别带 来的影响, 该框架使用并行网络分别学习 RoI 和全 局区域的特征, 并连接这些并行层的特征向量, 得 到混合特征用于微表情识别.

\section{1 相关工作}

最早期的微表情识别工作是基于局部二进制 模式(local binary pattern, LBP)展开的, Zhao 等 ${ }^{[4]}$ 将 LBP 从二维空间扩展到三维空间, 得到三正交平 面局部二进制特征(local binary patterns from three orthogonal planes, LBP-TOP), 并使用多核学习对 微表情进行分类. 此后, LBP-TOP 又被扩展到张量 独立的色彩空间中以获得更鲁棒的子空间 ${ }^{[2]}$.

现有方法除了考虑微表情的空间信息，还考 虑序列间的运动信息.微表情序列信息的提取有 2 种. (1) 从光流中提取出运动信息. Liong 等 ${ }^{[5]}$ 提出 的特征抽取器——双加权定向光流 (bi-weighted oriented optical flow, Bi-WOOF), 可以根据光流自 身的大小和光学应变的大小进行局部加权得到光 流直方图特征, 用于描述微表情的运动特征. Gan 等 ${ }^{[23]}$ 利用顶点帧和起始帧获得光流信息, 并将光流 特征输人预先设计的卷积神经网络(convolutional neural networks, CNN)中, 以进一步增强特征并进 行微表情分类. Liong 等 ${ }^{[9]}$ 利用浅层三流三维神经 网络(shallow triple stream three-dimensional CNN, STSTNet)对微表情序列的 3 种光流信息(光学应变 特征、水平光流特征和垂直光流特征)进行特征提 取融合, 并利用融合特征对微表情识别. 与之相 


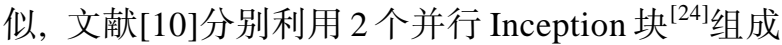
的网络提取水平光流和垂直光流的特征图, 然后 将 2 个特征图融合, 继续学习并分类. Liu 等 ${ }^{[8]}$ 也进 行了从宏表情光流信息到微表情光流信息迁移学 习的工作. 这些工作都利用光流对运行信息进行 建模, 分为 2 个步骤: 首先需要单独计算出光流信 息并保存下来，然后将光流信息作为神经网络的 输人进行训练识别, 因此操作烦琐, 并不能达到端 到端的识别. (2) 利用 CNN 和长短时记忆网络(long short-term memory networks, LSTM)结合的方式建 模运动随时间变化的关系. Choi 等 ${ }^{[25]}$ 提出一种基 于人脸地标特征图的情感识别方法, 其是 $\mathrm{CNN}+$ LSTM 的集成框架, 将二维人脸地标点转化为二维 图像作为输人, 进行微表情识别. Wang 等 ${ }^{[6]}$ 也采用 CNN+LSTM 框架学习微表情序列的时空信息, 但 其中增加了网络参数, 需要消耗更多的计算资源. 目前, 自发式微表情的数据集样本较少, 过多的网 络参数容易造成过拟合的现象. 针对以上 2 种视频 序列信息提取方法的不足, Jiang 等 ${ }^{[26]}$ 在动作识别任 务中提出以二维卷积网络为主干的框架, 其中利用 时空模块(spatio-temporal module, STM)并行地对时 空信息和运动信息建模时空特征和运动特征，最后 将两者融合在一起. 不同于 STM, 本文利用运动特 征提取模块计算相邻空间特征图的差值, 以得到运 动特征, 并将这些特征和空间特征叠加得到时空混 合特征，提高特征图的质量，改善网络的可分辨性.

还有许多关于微表情识别的方法. Ben 等 ${ }^{[27]}$ 通 过最大化类间拉普拉斯散射和最小化类内拉普拉 斯散射寻找张量到张量投影, 直接从原始张量数 据中提取出具有区分性的几何保留特征. Xia 等 ${ }^{[28]}$ 提出一种递归卷积网络，通过探索浅层网络结构 和较低分辨率的输人样本提高识别性能. 文献 [29] 首次将遗传算法应用到微表情识别任务中, 利用 遗传算法消除那些微表情预测的无关信息来丰富 特征表达，通过将遗传算法集成到 STSTNet后，识 别性能得到了提升. 文献[11]将胶囊网络引用到微 表情识别中, 将顶点帧像素信息输人胶囊网络中 提取特征并分类. 这些微表情识别方法虽然取得 了一定的成功，但是并没有考虑到微表情帧之间 的运动信息。

\section{2 本文方法}

\section{1 视频预处理}

视频预处理即对微表情序列进行归一化操作,
方便神经网络学习. (1) 用 Haar 脸部检测器和主动 形状模型 ${ }^{[30]}$ 对人脸进行检测并定位 68 个地标点. 为了确保人脸水平端正, 通过判断两眼眼角地标 点是否在一条水平线上进行旋转调整, 直至水平. (2) 根据人脸地标点切分出人脸区域. (3) 对微表 情帧序列进行灰度化操作, 减少深度模型学习的 参数. 处理结果如图 1 所示.

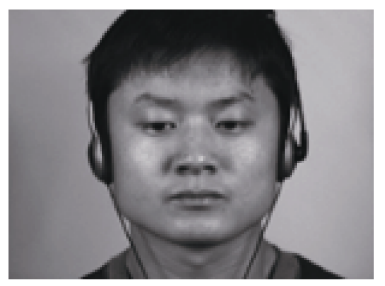

a. 原始图片

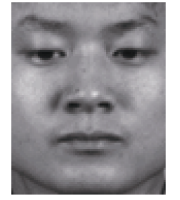

b. 处理后的图片

图 1 人脸切分

\subsection{RoI 划分}

微表情的产生存在于局部区域 ${ }^{[14]}$ (主要集中在 眼睛、眉毛和嘴部), 尽管全脸图像包含了微表情 的全部信息，但是 RoI 信息也具有 3 个优势:

(1) 删除与微表情无关或弱相关区域, 聚焦研 究局部细节信息;

（2）避免无效区域的背景噪声干扰，提高识别 性能;

(3) 减少输人的数据量和计算时间, 提高计算 性能.

自发式微表情数据集 ${ }^{\left[{ }^{[31-32]}\right.}$ 在定义情感表情类 别时是根据 AUs 进行划分的, 这些单元主要集中 在眼睛、眉毛、嘴、脸颊以及鼻子的周围. 表 1 所 示为这些 RoI, AUs, 情感类别以及 AUs 在数据集 中出现的频次. 可以看出, 鼻子和脸爽区域的 AUs 出现频次远低于眉毛+眼睛和嘴部区域. 因此, 为 了提高数据的信噪比, 本文舍弃相对不活跃的区 域，选择了眉毛+眼睛和嘴部作为 RoI.

表 1 RoI, AUs 情感类别和频次之间的关系

\begin{tabular}{lllc}
\hline \multicolumn{1}{c}{ RoI } & \multicolumn{1}{c}{ AUs } & \multicolumn{1}{c}{ 情感类别 } & 频次 \\
\hline 眉毛+眼睛 & $1,2,4,7$ & $\begin{array}{l}\text { 悲伤, 庈恶, 惊奇, } \\
\text { 紧张, 恐惧 }\end{array}$ & 308 \\
嘴部 & $10,12,14,15,25$ & $\begin{array}{l}\text { 厌恶, 紧张, 惊奇, } \\
\text { 压抑, 郭视, 高兴 }\end{array}$ & 155 \\
鼻子 & 9 & 厌恶 & 18 \\
脸颊 & 6 & 高兴 & 18 \\
\hline
\end{tabular}

确定 RoI 之后，本文利用一种自动划分策略对 裁剪好的微表情帧进行 RoI划分. 首先将序列中的 每帧归一化到 $120 \times 140$ 像素, 此时微表情可划分 为长和宽均为 10 像素的 $12 \times 14$ 个小块; 然后垂直 
方向以 10 50 像素、水平方向以 0 120 像素为范围 截取眼睛+眉毛区域, 垂直方向以 90 120 像素、水 平方向以 20 100 像素截取嘴部区域, 如图 2 所示.

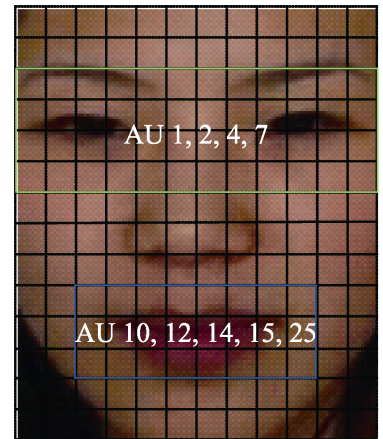

眼睛+眉毛

图 2 RoI 和 AUs 之间的对应关系

\section{3 运动特征提取}

微表情运动特征提取模块如图 3 所示. 不同于 光流计算帧之间像素级运动特征，该模块可以提 取空间特征图在时间维度上的特征级运动特征, 避免了额外计算开销且更加关注动作交互的部分.

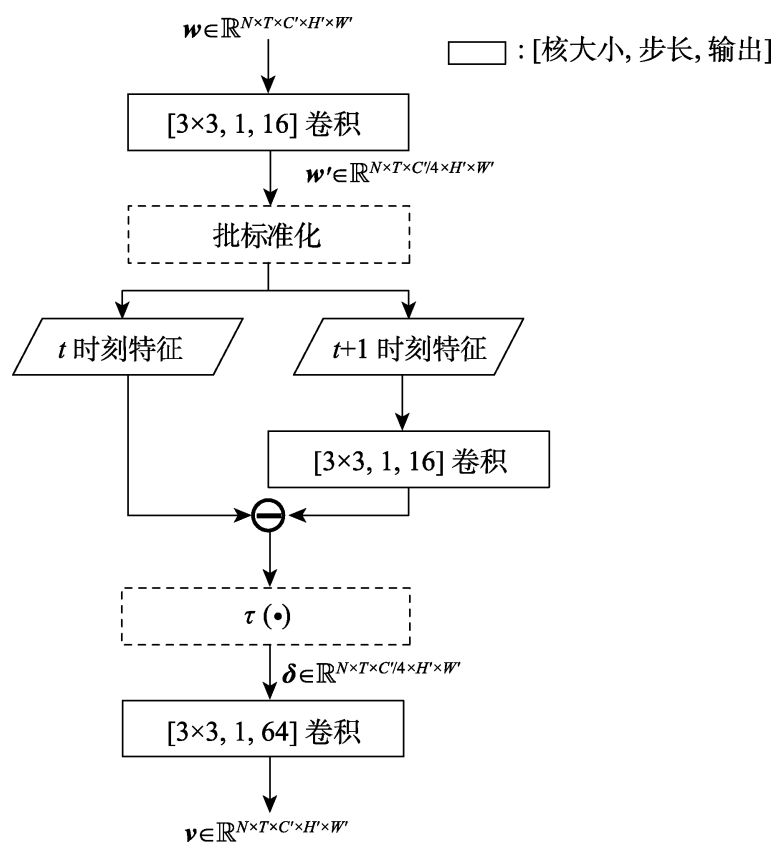

图 3 微表情运动特征提取模块

运动特征提取模块的输人是空间特征图 $\boldsymbol{w} \in \mathbb{R}^{N \times T \times C^{\prime} \times H^{\prime} \times W^{\prime}}$. 其中, $N$ 为训练批次的大小, $T$ 为每个微表情序列的帧数, $C^{\prime}$ 为特征图的数量, $H^{\prime}$ 和 $W^{\prime}$ 分别为特征图的长和宽. 在提取运动特 征之前, 先对特征图 $\boldsymbol{w}$ 进行通道压缩, 本文中相 对输人缩小了 4 倍, 公式为

$$
\boldsymbol{w}^{\prime}=f^{*}(\boldsymbol{w}), \boldsymbol{w}^{\prime} \in \mathbb{R}^{N \times T \times C^{\prime} / 4 \times H^{\prime} \times W^{\prime}}
$$

其中, $f^{*}(\cdot)$ 表示卷积核大小为 $3 \times 3$ 的卷积层. 由 于在微表情序列中后一帧是前一帧因发生空间位 移而得到的, 运动会使相邻帧中的对象发生空间 位移，会导致运动信息不匹配，从而无法直接计算 相邻帧位移变化特征. 为了解决这个问题, 模块中 在 $t+1$ 帧处添加了 $3 \times 3$ 卷积, 从上下文中捕获运动 对象的匹配区域. 然后逐时求差, 计算出前后帧之 间短时运动信息

$$
\boldsymbol{\delta}=\tau\left(f\left(\boldsymbol{w}^{\prime t+1}\right)-f\left(\boldsymbol{w}^{\prime t}\right)\right), 1<t<T-1
$$

其中, $f(\cdot)$ 表示二维卷积操作; $t$ 为微表情序列的 帧编号. 由于逐时差值后在时间维度会由 $T$ 变为 $T-1$, 因此利用 $\tau(\cdot)$ 进行补零. 最后对运动特征进 行解码得到运动信息

$$
\boldsymbol{v}=f^{\prime}(\boldsymbol{\delta}), \boldsymbol{v} \in \mathbb{R}^{N \times T \times C^{\prime} \times H^{\prime} \times W^{\prime}}
$$

其中, $f^{\prime}(\cdot)$ 表示核大小为 $3 \times 3$ 的卷积层.

\section{4 微表情特征提取与识别}

由于微表情变化幅度小，相邻帧之间的运动 特征不明显且存在大量的圥余信息, 在将微表情 序列输人神经网络前, 先采用稀疏时间采样法从 微表情序列中提取出稀疏序列，使相邻帧之间的 运动信息相对明显. 具体地, 将微表情序列均匀分 为 $T$ 个片段并从每个片段随机选取一帧，便可得到 长度为 $T$ 的微表情的序列, 作为神经网络的输人.

微表情识别框架如图 4 所示, 它由 3 个并行网 络组成，每个分支都使用相同的空间特征提取模 块对输人的局部或全局序列进行学习, 得到空间 特征图 $\boldsymbol{w} \in \mathbb{R}^{N \times T \times C^{\prime} \times H^{\prime} \times W^{\prime}}$ ，具体的参数设置如图 5 所示. 首先将 $\boldsymbol{w}$ 输人运动信息提取模块中, 以捕获 特征图的边缘运动信息, 进而得到特征级运动特征 $\boldsymbol{v} \in \mathbb{R}^{N \times T \times C^{\prime} \times H^{\prime} \times W^{\prime}}$. 然后将 $\boldsymbol{w}$ 和 $\boldsymbol{v}$ 进行聚合，即

$$
\boldsymbol{\varphi}=\boldsymbol{v}+\boldsymbol{w}, \boldsymbol{A} \in \mathbb{R}^{N \times T \times C^{\prime} \times H^{\prime} \times W^{\prime}}
$$

其中, $C^{\prime}$ 为空间特征图通道的数量; $H^{\prime}$ 和 $W^{\prime}$ 分别为 空间特征图的宽和高. 接着将 $\boldsymbol{\varphi}$ 送人全连接层学 习得到 $\lambda \in \mathbb{R}^{N \times T \times C}$, 并在时间维度上对特征取平均 值，即可得到微表情视频的特征，即

$$
\gamma=\sum_{t=1}^{T} \lambda(t), \gamma \in \mathbb{R}^{N \times D}
$$

其中, $D$ 为全连接层的输出维度, $D=1024$. 此时, 得到仅是一个分支的视频特征, 还需要将 3 个并行 网络的输出通过连接层合成一个综合的视频特征

$$
\boldsymbol{z}=\left\{\gamma_{\mathrm{m}} ; \gamma_{\mathrm{c}} ; \gamma_{\mathrm{h}}\right\}
$$

其中, $\gamma_{\mathrm{m}}, \gamma_{\mathrm{c}}, \gamma_{\mathrm{h}}$ 分别为从嘴部、眼睛+眉毛和整张 


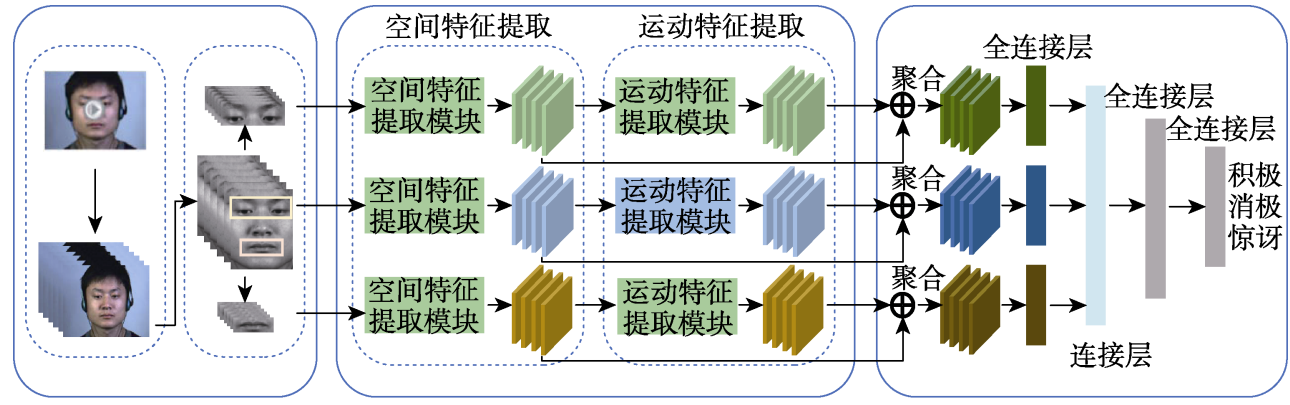

图 4 微表情识别框架

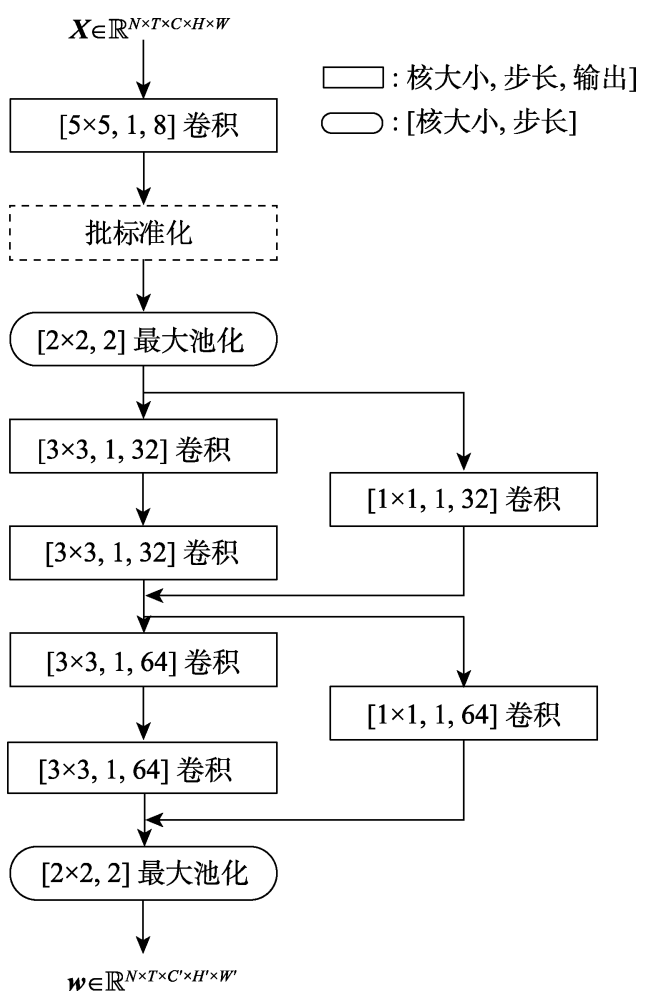

图 5 空间特征提取模块

脸中提取出来的特征. 最后将 $\boldsymbol{z}$ 送人 2 层全连接层 (维度分别取 1024 和 3)以及一个 Softmax 层得到识 别特征 $\boldsymbol{Z} \in \mathbb{R}^{N \times 3}$, 用于微表情的识别. 在 2 个连接 层之间还设置了一个丢包率为 0.5 的 dropout 层.

\section{3 实 验}

\section{1 数据集}

实验中使用 3 个自发式数据集：CASME II, SMIC 和 SAMM.

\subsubsection{CASME II 数据集}

CASME II 数据集 ${ }^{[32]}$ 包含 26 位中国志愿者的 247 个微表情序列, 样本示例如图 6a 所示. 该数据 集使用帧率为 200 帧/s 的摄像机进行采集，图片分 辨率为 $640 \times 480$ 像素; 提供的信息包括开始帧、顶 点帧、结束帧、情感标签和 AUs; 情感标签有幸福、
厌恶、惊奇、压抑和其他 5 种.

\subsubsection{SMIC 数据集}

图 6b 所示为 SMIC 数据集 ${ }^{[33]}$ 中包含的样本示 例图. 该数据集包含 164 个微表情序列, 来自 16 位志愿者 (8 位亚洲人, 8 位高加索人); 采样帧率为 25 帧 $/ \mathrm{s}$, 分辨率为 $640 \times 480$ 像素; 提供的信息有情 感标签、起始帧和结束帧; 情感标签为积极、消极 和惊讶.

\subsubsection{SAMM 数据集}

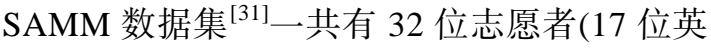
国白人, 3 位中国人, 2 位阿拉伯人, 2 位马来人, 非 洲人、非洲加勒比海地区人、英国非洲裔人、阿拉 伯人、印度人、尼泊尔人、巴基斯坦人和西班牙人 各 1 位), 每位志愿者各采集 7 条微表情序列，一共 224 条. 使用带灰度传感器的摄像机采集数据集, 帧率为 200 帧 $/ \mathrm{s}$, 分辨率为 $2040 \times 1088$ 像素. 该数 据集提供了微表情序列的开始帧、顶点帧、结束帧、 情感标签和 AUs. 情感标签有生气、悲伤、害怕、 鄙视、厌恶、开心、惊讶和其他. SAMM 数据集的 样本示例如图 6c 所示.

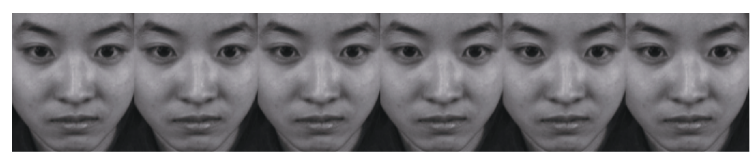

a. CASME II ${ }^{[32]}$

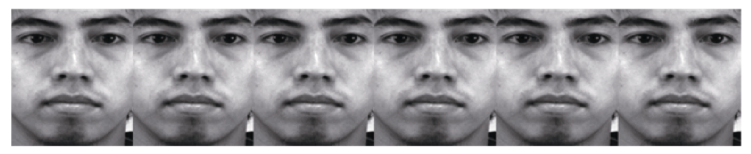

b. SMIC ${ }^{[33]}$

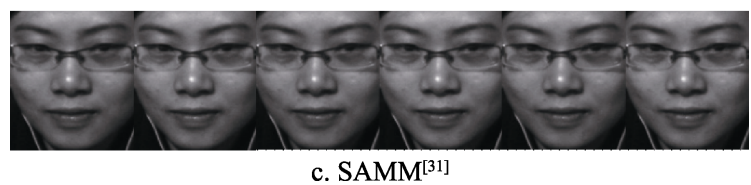

图 63 个数据集惊讶标签示例图

数据集样本的具体分布如表 2 所示. 可以看 出, 这些自发式微表情数据集的样本数量不足, 利 用神经网络识别微表情很容易造成过拟合问题. 
针对这一问题, 本文采用第 2 届微表情识别大赛 (MEGC 2019)数据集 ${ }^{[34]}$, 其将 3 个自发式微表情数 据集(SAMM, CASME II 和 SMIC)集成到单个交叉 数据集中. 集成后的数据集较单个数据集增加了 视频样本总数, 更加契合以数据为驱动的深度学 习方法. 此外, 将原有的多种情感类别简化到一般 情感类别, 能更好地避免因不同刺激和环境设置 所引发的情感类别的模糊性. 在简化情感类别时, MEGC2019 将原有的标签重新映射到新的标签空 间 $\{$ 积极、消极、惊讶 $\}$ 中，具体映射关系如下：

(1) 消极. \{压抑、生气、鄙视、厌恶、害怕、 伤心、消极 $\}$;

(2) 积极. \{开心、积极 $\}$;

(3) 惊讶. $\{$ 惊讶 $\}$.

表 2 数据集的样本分布

\begin{tabular}{lcccc}
\hline \multicolumn{1}{c}{ 数据集名称 } & 积极 & 消极 & 惊讶 & 合计 \\
\hline CASME II $^{[32]}$ & 32 & $88^{*}$ & 25 & 145 \\
SMIC $^{[33]}$ & 51 & 70 & 43 & 164 \\
SAMM $^{[31]}$ & 26 & $92^{* *}$ & 15 & 133 \\
MEGC 2019 $^{[34]}$ & 109 & 250 & 83 & 442 \\
\hline
\end{tabular}

注. *仅包含厌恶和压抑; **仅包含生气、鄙视、厌恶、害 怕和伤心

\section{2 实验评估标准}

实验采用留一法进行交叉验证，即每位志愿 者的情感视频都将单独作为测试集，其余志愿者 的情感视频均作为训练集, 迭代交叉验证 $k$ 次, $k$ 为数据集中的志愿者的人数. MEGC 2019 中总共 有 68 位志愿者(包含 SMIC 的 16 位、CASME II 的 24 位以及 SAMM 的 28 位), 因此取 $k=68$.

从表 2 可以看出, 3 个类别的样本数量不平衡. 为此, 文献[35]设计了 2 个指标来评估实验的性能, 分别是未加权 $F_{1}$ 分数( unweighted $F_{1}$-score, $\mathrm{UF}_{1}$ ) 和 未加权平均召回率 (unweighted average recall, $U A R$ ). 计算 $U_{1}$ 时, 首先统计真阳性(true positive, TP)、假阳性 (false positive, FP)、假阴的 (false negative, $\mathrm{FN}$ )的个数, 计算每类各自的 $F_{1}$ 分数 $\left(F_{1}\right.$-score, $\left.\mathrm{FS}\right)$ 、准确率 $P$ 和召回率 $R$; 然后将所有 类的 FS 取均值便可得到 $\mathrm{UF}_{1}$. 计算公式分别为

$$
\begin{gathered}
\mathrm{FS}=\frac{2 \times P_{c} \times R_{c}}{P_{c}+R_{c}} \\
P_{c}=\frac{\sum_{\sigma=1}^{k} \mathrm{TP}_{c}^{\sigma}}{\sum_{\sigma=1}^{k} \mathrm{TP}_{c}^{\sigma}+\sum_{\sigma=1}^{k} \mathrm{FP}_{c}^{\sigma}}
\end{gathered}
$$

$$
R_{c}=\frac{\sum_{\sigma=1}^{k} \mathrm{TP}_{c}^{\sigma}}{\sum_{\sigma=1}^{k} \mathrm{TP}_{c}^{\sigma}+\sum_{\sigma=1}^{k} \mathrm{FN}_{c}^{\sigma}}
$$

其中, $c$ 为标签类别, $k$ 为志愿者的数量, $\mathrm{TP}_{c}$ 为 $c$ 识别正确的样本数量, $\mathrm{FP}_{c}$ 为将其他类别识别为 $c$ 的样本数量, $\mathrm{FN}_{c}$ 为将 $c$ 识别为其他类别的数量. 因此, $\mathrm{UF}_{1}$ 可以表示为

$$
\mathrm{UF}_{1}=\frac{1}{C} \sum_{c=1}^{C} \mathrm{FS}_{c}
$$

其中, $C$ 为微表情的标签数量.

UAR 度量标准也称为系统的平衡精度. 对于 样本不均衡的数据集, 分类器很难从样本数量较 少的类别中拟合参数, 而 UAR 可能会偏向类别数 量较大的分类器, 进而全面分析分类器的性能. 计 算UAR 之前, 先计算每个类别的识别正确率 $A$, 即

$$
A_{c}=\frac{\sum_{\sigma=1}^{k} \mathrm{TP}_{c}^{\sigma}}{N_{c}}
$$

其中, $N_{c}$ 为类 $c$ 的样本数量; 然后对所有类别的 $A_{c}$ 取均值得到 UAR, 即

$$
\mathrm{UAR}=\frac{1}{C} \sum_{c=1}^{C} A_{c}
$$

\section{3 实验结果与分析}

本节评估局部特征对微表情识别的影响，分 析微表情识别中稀疏采样帧与 UAR 之间的关系, 最后与其他工作进行对比.

\subsection{1 局部与全局特征识别结果}

表 3 所示为利用局部特征、全局特征和串联特 征的识别结果. 其中, 局部特征(眼睛+眉毛特征、 嘴部特征)是以对应 RoI 作为输人得到的特征, 全 局特征是以整张人脸作为输人得到特征, 将所有 的 RoI 和整个人脸输人并行网络后所提取到的特 征通过连接层和全连接层得到串联特征, 如图 4 所 示. 可以看出, 以全局特征的识别结果作为基线, 眼+眉毛和嘴部的结果低于基线, 这是因为基线的 识别结果是使用包含所有 RoI 的整张人脸提取得 到的, 而在 MEGC 2019 数据集上, 将全脸特征和 所有 RoI 特征相结合后的 UAR 结果比基线高了 $2.72 \%$. 该结果表明, RoI 特征可以弥补因学习全局 特征而忽略的一些局部特征，同时还可以避免不 活跃区域带来的噪声干扰. 即 RoI 和全局区域包含 着的是不同水平的信息, 将细节和整体信息融合 能够取得更好的识别结果. 
表 3 全局特征、局部特征和串联特征的识别率

\begin{tabular}{|c|c|c|c|c|c|c|c|c|}
\hline \multirow{2}{*}{ 区域特征 } & \multicolumn{2}{|c|}{ CASME II $^{[32]}$} & \multicolumn{2}{|c|}{$\mathrm{SMIC}^{[33]}$} & \multicolumn{2}{|c|}{$\mathrm{SAMM}^{[31]}$} & \multicolumn{2}{|c|}{ MEGC $2019^{[34]}$} \\
\hline & $\mathrm{UF}_{1}$ & UAR & $\mathrm{UF}_{1}$ & UAR & $\mathrm{UF}_{1}$ & UAR & $\mathrm{UF}_{1}$ & UAR \\
\hline 眼睛+眉毛特征 & 0.7666 & 0.7843 & 0.6936 & 0.6856 & 0.7287 & 0.7745 & 0.7698 & 0.7468 \\
\hline 嘴部特征 & 0.7782 & 0.7524 & 0.7082 & 0.6921 & 0.7496 & 0.7443 & 0.7621 & 0.7524 \\
\hline 全局特征 & 0.7939 & 0.7931 & 0.7355 & 0.7149 & 0.7675 & 0.7929 & 0.7751 & 0.7629 \\
\hline 串联特征(本文) & 0.8584 & 0.8569 & 0.7573 & 0.7559 & 0.7873 & 0.8221 & 0.8181 & 0.7901 \\
\hline
\end{tabular}

注. 粗体表示最佳实验结果.

\subsection{2 时间稀疏采样识别结果}

微表情变化微弱，帧之间的运动变化不太明 显，存在大量冗余信息. 针对该问题，本文使用时 间稀疏采样策略, 在避免数据冗余的同时保证帧 之间的运动相对明显, 进而对微表情进行有效的 运动建模. 图 7 所示为稀疏采样帧的数量与 UAR 间的关系, 可以看出, 在 4 个数据集中, 稀疏采样 帧数量为 6 时的 UAR 高于取 7 帧和 5 帧的结果. 这 说明当采样帧不足时，微表情序列的信息不全，提 取到的微表情特征不具有完整性. 另外, 当达到一 定帧时，微表情序列中的信息量已经达到饱和，此 时再加人新帧不但没有提升性能，反而会引人更 多的噪声，进而对识别造成干扰.

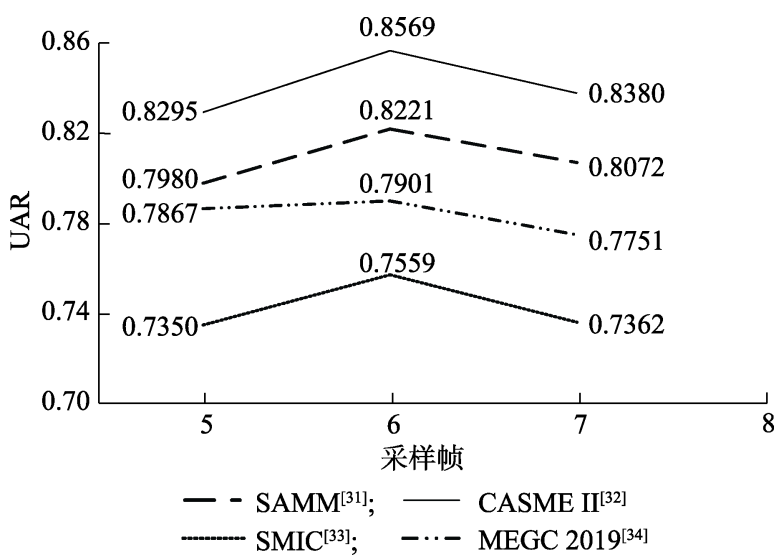

图 7 采样帧数与 UAR 之间的关系

\subsection{3 与其他方法的对比}

表 4 所示为本文方法与其他最新方法的比较 的结果, 所有的方法都使用了相同的数据集和验 证方法. 可以看出, 除了 CASME II 数据集, 本文 方法在其他数据集上的 UAR 和 $\mathrm{UF}_{1}$ 都获得了最好 的结果; 相对于其他方法只提取单一的空间特征 或运动特征用于微表情识别, 或者仅对微表情序 列的顶点帧进行识别, 本文对微表情序列分别提 取了这 2 个特征并将两者聚合成新的特征再去识别, 由于空间特征包含面部外观信息, 而运动特征包又 含随时间变化的运动信息, 将这 2 个不同类型的特 征互补聚合，可以形成更具有区分性的新特征.

图 8 所示为在 4 个数据集上识别的混淆矩阵. 可以看出, 在消极微表情的识别上都取得了最高 的识别率; 另外, 在每个数据集的错误识别中, 大 部分都识别为消极微表情, 这可能是数据集中消 极样本占据大部分而导致样本不均衡所致的. 但 是, 任意数据集的每个类别的正确识别数量都大 于错误识别数量, 这证实了本文方法的有效性.

\section{4 结 语}

本文提出一种端到端的微表情识别方法. 首 先用残差神经网络提取微表情的空间特征, 然后 利用动作提取模块提取空间特征的特征级的动作

表 4 与现有方法性能的比较

\begin{tabular}{|c|c|c|c|c|c|c|c|c|}
\hline \multirow{2}{*}{ 方法 } & \multicolumn{2}{|c|}{${\text { CASME } \text { II }^{[32]}}$} & \multicolumn{2}{|c|}{$\mathrm{SMIC}^{[33]}$} & \multicolumn{2}{|c|}{ SAMM $^{[31]}$} & \multicolumn{2}{|c|}{ MEGC 2019 ${ }^{[34]}$} \\
\hline & $\mathrm{UF}_{1}$ & UAR & $\mathrm{UF}_{1}$ & UAR & $\mathrm{UF}_{1}$ & UAR & $\mathrm{UF}_{1}$ & UAR \\
\hline LBP-TOP $^{[4]}$ & 0.7026 & 0.7429 & 0.2000 & 0.5280 & 0.3954 & 0.4102 & 0.5882 & 0.5785 \\
\hline $\mathrm{Bi}-\mathrm{WOOF}^{[5]}$ & 0.7805 & 0.8026 & 0.5727 & 0.5829 & 0.5211 & 0.5139 & 0.6296 & 0.6227 \\
\hline OFF-ApexNet ${ }^{[23]}$ & 0.8764 & 0.8681 & 0.6817 & 0.6695 & 0.5409 & 0.5392 & 0.7196 & 0.7096 \\
\hline CapsuleNet $^{[11]}$ & 0.7068 & 0.7018 & 0.5820 & 0.5877 & 0.6209 & 0.5989 & 0.6520 & 0.6506 \\
\hline Dual-Inception $^{[10]}$ & 0.8621 & 0.8560 & 0.6645 & 0.6726 & 0.5868 & 0.5663 & 0.7322 & 0.7278 \\
\hline STSTNet $^{[9]}$ & 0.8382 & 0.8686 & 0.6801 & 0.7013 & 0.6588 & 0.6810 & 0.7353 & 0.7605 \\
\hline $\mathrm{EMR}^{[8]}$ & 0.8293 & 0.8209 & 0.7461 & 0.7530 & 0.7754 & 0.7152 & 0.7885 & 0.7824 \\
\hline $\mathrm{RCN}^{[28]}$ & 0.8087 & 0.8563 & 0.5980 & 0.5991 & 0.6771 & 0.6976 & 0.7052 & 0.7164 \\
\hline $\operatorname{LFM}^{[25]}$ & 0.8700 & 0.8400 & 0.7200 & 0.7100 & 0.6700 & 0.6000 & 0.7700 & 0.7500 \\
\hline STSTNet+GA ${ }^{[25,29]}$ & 0.8872 & 0.8872 & 0.7169 & 0.7438 & 0.6678 & 0.6995 & 0.7663 & 0.7885 \\
\hline 本文 ～～～～～ & 0.8584 & 0.8569 & 0.7573 & 0.7559 & 0.7873 & 0.8221 & 0.8181 & 0.7901 \\
\hline
\end{tabular}

注. 粗体表示最佳实验结果. 


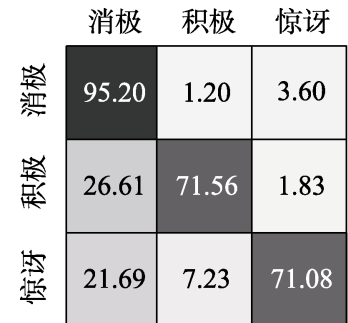

a. MEGC 2019[34]

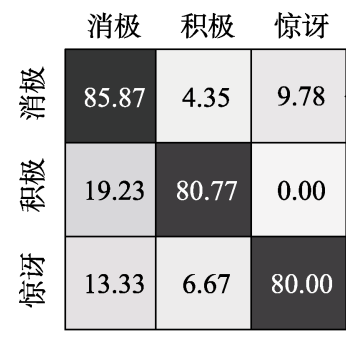

c. $\mathrm{SAMM}^{[31]}$

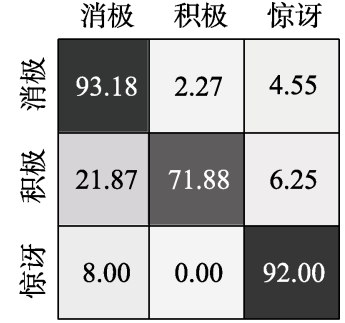

b. CASME $\Pi^{[32]}$

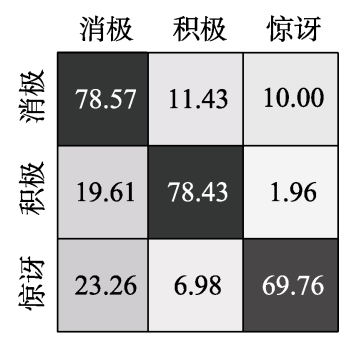

d. $\mathrm{SMIC}^{[33]}$
图 8 混淆矩阵

特征，再将两者聚合，丰富微表情特征，提高网络 的识别性能. 采用 MEGC 2019 数据集以及评测标 准进行实验的结果证明, 本文方法比许多现有的 方法取得了更好的识别性能; 另外，通过实验还验 证了 RoI 对于微表情识别的影响. 本文根据微表情 AUs 的活跃频次选取 RoI，分别利用并行网络提取 全局和 RoI 特征. 实验结果表明, 2 个不同层次的 拼接特征的识别结果优于单一特征的识别结果. 在动作特征提取时，本文考虑相邻帧之间的特征 提取, 即短时的运动特征. 未来的工作会聚焦在提 取到长时运动特征，即连续帧之间的特征，以提高 识别性能; 本文方法在 CASME II 数据集的实验结 果不是最优，这可能是由于没有考虑少部分活跃 区域的原因，今后将充分研究微表情识别与活跃 区域之间的关系. 另外, 微表情数据样本不平衡带 来的决策边界偏移问题还有待解决.

\section{参考文献(References):}

[1] Ben Xianye, Yang Mingqiang, Zhang Peng, et al. Survey on automatic micro-expression recognition methods[J]. Journal of Computer-Aided Design \& Computer Graphics, 2014, 26(9): 1385-1395 (in Chinese) (贲晛烨，杨明强，张鹏，等. 微表情自动识别综述 [J]. 计算 机辅助设计与图形学学报, 2014, 26(9): 1385-1395)

[2] Ekman P, Friesen W V. Nonverbal leakage and clues to deception[J]. Psychiatry, 1969, 32(1): 88-106

[3] Sharon W. Airport security: intent to deceive?[J]. Nature, 2010, 465(7297): 412-415

[4] Zhao G Y, Pietikäinen M. Dynamic texture recognition using local binary patterns with an application to facial expres-
sions[J]. IEEE Transactions on Pattern Analysis and Machine Intelligence, 2007, 29(6): 915-928

[5] Liong S T, See J, Wong K S, et al. Less is more: micro-expression recognition from video using apex frame[J]. Signal Processing: Image Communication, 2018, 62: 82-92

[6] Wang S J, Li B J, Liu Y J, et al. Micro-expression recognition with small sample size by transferring long-term convolutional neural network[J]. Neurocomputing, 2018, 312: 251-262

[7] Liu Ruhan, Xu Dan. Video amplification and deep learning in micro-expression recognition[J]. Journal of Computer-Aided Design \& Computer Graphics, 2019, 31(9): 1535-1541(in Chinese)

(刘汝涵, 徐丹. 视频放大和深度学习在微表情识别任务上 的应用[J]. 计算机辅助设计与图形学学报, 2019, 31(9): 1535-1541)

[8] Liu Y C, Du H M, Zheng L, et al. A neural micro-expression recognizer[C] //Proceedings of the 14th IEEE International Conference on Automatic Face and Gesture Recognition. Los Alamitos: IEEE Computer Society Press, 2019: Article No.8756583

[9] Liong S T, Gan Y S, See J, et al. Shallow triple stream three-dimensional CNN (STSTNet) for micro-expression recognition[C] //Proceedings of the 14th IEEE International Conference on Automatic Face and Gesture Recognition. Los Alamitos: IEEE Computer Society Press, 2019: Article No.8756567

[10] Zhou L, Mao Q R, Xue L Y. Dual-Inception network for cross-database micro-expression recognition[C] //Proceedings of the 14th IEEE International Conference on Automatic Face and Gesture Recognition. Los Alamitos:IEEE Computer Society Press, 2019: Article No.8756579

[11] Van Quang N, Chun J, Tokuyama T. CapsuleNet for micro-expression recognition[C] //Proceedings of the 14th IEEE International Conference on Automatic Face and Gesture Recognition. Los Alamitos: IEEE Computer Society Press, 2019: Article No.8756544

[12] Lucas B D, Kanade T. An iterative image registration technique with an application to stereo vision[C] //Proceedings of the 7th International Joint Conference on Artificial Intelligence. San Francisco: Morgan Kaufmann Publishers Inc. Press, 1981: 674-679

[13] Ji S W, Xu W, Yang M, et al. 3D Convolutional neural networks for human action recognition[J]. IEEE Transactions on Pattern Analysis and Machine Intelligence, 2013, 35(1): 221-231

[14] Allaert B, Bilasco I M, Djeraba C. Micro and macro facial expression recognition using advanced local motion patterns [J/OL]. IEEE Transactions on Affective Computing: 1-12 [2020-10-19]. https://doi.org/10.1109/TAFFC.2019.2949559

[15] Ekman P, Friesen W V. Facial action coding system (FACS): a technique for the measurement of facial actions[J]. Rivista Di Psichiatria, 1978, 47(2): 126-138

[16] Happy S L, Routray A. Automatic facial expression recognition using features of salient facial patches[J]. IEEE Transactions on Affective Computing, 2015, 6(1): 1-12

[17] Lucey P, Cohn J F, Kanade $\mathrm{T}$, et al. The extended 
Cohn-Kanadedataset (CK+): a complete dataset for action unit and emotion-specified expression[C] //Proceedings of the IEEE Computer Society Conference on Computer Vision and Pattern Recognition Workshops. Los Alamitos: IEEE Computer Society Press, 2010: 94-101

[18] Lyons M, Kamachi M, Gyoba J. The Japanese female facial expression (JAFFE) dataset[OL]. [2020-10-19]. https://zenodo. org/record/3451524

[19] Ringeval F, Schuller B, Michel V, et al. AV+EC 2015: the first affect recognition challenge bridging across audio, video, and physiological data[C] //Proceedings of the 5th International Workshop on Audio/Visual Emotion Challenge. New York: ACM Press, 2015: 3-8

[20] Yan W J, Wang S J, Chen Y H, et al. Quantifying micro-expressions with constraint local model and local binary pattern[C] //Proceedings of the European Conference on Computer Vision. Heidelberg: Springer, 2015: 296-305

[21] Wang S J, Yan W J, Zhao G Y, et al. Micro-expression recognition using robust principal component analysis and local spatiotemporal directional features[C] //Proceedings of the European Conference on Computer Vision. Heidelberg: Springer, 2014: 325-338

[22] Wang S J, Yan W J, Li X B, et al. Micro-expression recognition using color spaces[J]. IEEE Transactions on Image Processing, 2015, 24(12): 6034-6047

[23] Gan Y S, Liong S T, Yau W C, et al. OFF-Apex net on micro-expression recognition system[J]. Signal Processing: Image Communication, 2019, 74: 129-139

[24] Szegedy C, Liu W, Jia Y Q, et al. Going deeper with convolutions[C] //Proceedings of the IEEE Conference on Computer Vision and Pattern Recognition. Los Alamitos: IEEE Computer Society Press, 2015: 1-9

[25] Choi D Y, Song B C. Facial micro-expression recognition using two-dimensional landmark feature maps[J]. IEEE Access, 2020, 8: 121549-121563

[26] Jiang B Y, Wang M M, Gan W H, et al. STM: spatio-temporal and motion encoding for action recognition[C] //Proceedings of the IEEE International Conference on Computer Vision.
Los Alamitos: IEEE Computer Society Press, 2019: 20002009

[27] Ben X Y, Zhang P, Yan R, et al. Gait recognition and micro-expression recognition based on maximum margin projection with tensor representation[J]. Neural Computing and Applications, 2016, 27(8): 2629-2646

[28] Xia Z Q, Peng W, Khor H Q, et al. Revealing the invisible with model and data shrinking for composite-database micro-expression recognition[J]. IEEE Transactions on Image Processing, 2020, 29: 8590-8605

[29] Jin Q S, Xu H C, Liu K H, et al. GA-ApexNet: genetic algorithm in apex frame network for micro-expression recognition system[J]. Journal of Physics: Conference Series, 2020, 1554: 012149

[30] Cootes T F, Taylor C J, Cooper D H, et al. Active shape models-their training and application[J]. Computer Vision and Understanding, 1995, 61(1): 38-59

[31] Davison A K, Lansley C, Costen N, et al. SAMM: a spontaneous micro-facial movement dataset[J]. IEEE Transactions on Affective Computing, 2018, 9(1): 116-129

[32] Yan W J, Li X B, Wang S J, et al. CASME II: an improved spontaneous micro-expression database and the baseline evaluation [J]. PLoS One, 2014, 9(1): e86041

[33] Li X B, Pfister $\mathrm{T}$, Huang X $\mathrm{H}$, et al. A spontaneous micro-expression database: inducement, collection and baseline[C] //Proceedings of the 10th IEEE International Conference and Workshops on Automatic Face and Gesture Recognition. Los Alamitos: IEEE Computer Society Press, 2013: Article No.6553717

[34] See J, Yap M H, Li J, et al. MEGC 2019 - the second facial micro-expressions grand challenge[C] //Proceedings of the 14th IEEE International Conference on Automatic Face and Gesture Recognition. Los Alamitos: IEEE Computer Society Press, 2019: 1-5.

[35] Ngo A C L, Phan C W, See J. Spontaneous subtle expression recognition: imbalanced databases and solutions[C] //Proceedings of the 12th Asian Conference on Computer Vision. Heidelberg: Springer, 2014: 33-48 